\title{
Genetic Diversity and Host Range Variation of Ralstonia solanacearum Strains Entering North America
}

\author{
David J. Norman, Mildred Zapata, Dean W. Gabriel, Y. P. Duan, Jeanne M. F. Yuen, \\ Arianna Mangravita-Novo, and Ryan S. Donahoo
}

First, fifth, and sixth authors: Department of Plant Pathology, University of Florida, IFAS, Mid-Florida Research and Education Center, 2725 Binion Rd., Apopka 32703; second author: Crop Protection Department, University of Puerto Rico, P.O. Box 9030, Mayaguez, Puerto Rico 00681; third author: Plant Molecular and Cellular Biology Program, Plant Pathology Department, University of Florida, Gainesville 32611; fourth author: United States Department of Agriculture-Agricultural Research Service (USDA-ARS)-USHRL, Fort Pierce, FL 34945; and seventh author: USDA-ARS USVL, Charleston, SC. Accepted for publication 18 April 2009.

\begin{abstract}
Norman, D. J., Zapata, M., Gabriel, D. W., Duan, Y. P., Yuen, J. M. F., Mangravita-Novo, A., and Donahoo, R. S. 2009. Genetic diversity and host range variation of Ralstonia solanacearum strains entering North America. Phytopathology 99:1070-1077.

Each year, large volumes of ornamental and food plant propagative stock are imported into the North America; occasionally, Ralstonia solanacearum is found systemically infecting this plant material. In this study, 107 new $R$. solanacearum strains were collected over a 10-year period from imported propagative stock and compared with 32 previously characterized $R$. solanacearum strains using repetitive polymerase chain reaction (rep-PCR) element (BOX, ERIC, and REP) primers. Additional strain comparisons were made by sequencing the endoglucanase and the

original host. Similarity coefficients among rep-PCR clusters within biovars were relatively low in many cases, indicating that disease outbreaks over time may have been caused by different clonal populations. Similar population differentiations of $R$. solanacearum were obtained when comparing strain sequences using either the endoglucanase or cytochrome $b 561$ genes. We found that most of the new biovar 1 strains of $R$. solanacearum entering the United States were genetically distinct from the biovar 1 strains currently found infecting vegetable production. These introduced biovar 1 strains also had a broader host range and could infect not only tomato, tobacco, and potato but also anthurium and pothos and cause symptoms on banana. All introductions into North America of race 3 , biovar 2 strains in the last few years have been linked to geranium production and appeared to be clonal.
\end{abstract} cytochrome $b 561$ genes. Using rep-PCR primers, populations could be distinguished by biovar and, to a limited extent, country of origin and
Additional keywords: bacterial wilt, FAME.
The United States Department of Agriculture estimates the yearly wholesale value of floricultural crops, including flowering, bedding, and tropical foliage, to be in excess of $\$ 5.2$ billion (36). Steady annual increases in sales have occurred in the floricultural industry. As this industry has grown, production has also diversified to include hundreds of plant species and cultivars. Many of these crops are labor intensive, specifically during early stages of production. As a result, much of the initial crop production is done in countries with low labor costs. In order to satisfy consumer's demands, over $\$ 568$ million worth of ornamental propagative materials are imported annually (38). Most of the imported floricultural propagative material enters the United States through Florida, California, and Texas. From these entry points, propagated material is rooted, grown, and shipped throughout the United States and North America. Production facilities contain plants from temperate, subtropical, and tropical origins. With introductions of so many new plant species, new pathogens are inadvertently introduced. Over the past 20 years, there have been many new pests described on floricultural crops and, as the industry continues to grow, this trend will undoubtedly continue.

Bacterial wilt caused by Ralstonia solanacearum (Smith 1896) Yabuuchi et al. 1996 (synonym Pseudomonas solanacearum) is a common bacterial disease in tropical and subtropical areas of the world. This pathogen is known to infect hundreds of plant species

Corresponding author: D. J. Norman; E-mail address: djn@ufl.edu

doi:10.1094/PHYTO-99-9-1070

(C) 2009 The American Phytopathological Society encompassing 44 families (9). Extensive damages to crops of banana, potato, tomato, and pepper production in tropical and subtropical regions are well documented. Most of the research conducted on bacterial wilt to date has been in relation to largeacreage agronomic crops, and recent research on geraniums has been limited $(31,42)$. Among many potential ornamental hosts, $R$. solanacearum has been described infecting anthurium, pothos, ornamental ginger, gerbera, zinnia, salvia, verbena, heliconia, sunflower, and geranium $(16,22,23,30,42)$.

$R$. solanacearum has historically been disseminated in banana and potato propagative material $(2,7,34)$. The potential movement of a systemic pathogen such as $R$. solanacearum in propagative material between countries has likely been underestimated. This potential mechanism of dissemination is even greater in cases where plants are not considered susceptible. Thus, theses hosts are not examined for particular pathogens. When new undescribed pathotypes of $R$. solanacearum are introduced from propagative material into a new environment, the potential impact on local crops in a new environment is unpredictable. Here, we compare populations of $R$. solanacearum entering North America on ornamental crop material using repetitive polymerase chain reaction (rep-PCR), amplified fragment length polymorphism (AFLP), sequence analysis of the endoglucanase and the cytochrome b561 genes, and host range.

\section{MATERIALS AND METHODS}

$R$. solanacearum strains were collected over a 10-year period. Plant samples were taken directly from imported boxes of 
cuttings, recently planted cuttings, and outbreaks within more established plantings. In all cases, host of origin and country of origin of plant material were obtained and recorded. Additional $R$. solanacearum strains were obtained from locations considered to have a higher probability of being introduced into North America due to proximity or agricultural trade. In addition, a number of reference strains along with strains isolated from solanaceous hosts in Florida were included for comparison (Table 1).

Bacteria were isolated from surface-sterilized $(0.3 \% \mathrm{NaOCl})$ leaves and stems of infected cuttings on modified Kelman's (21) triphenyltetrazolium chloride (TZC) medium. Individual colonies were selected and assessed for purity after incubation at $28^{\circ} \mathrm{C}$ for $48 \mathrm{~h}$. Selected colonies were mucoid and displayed the characteristic red swirling egg-shaped pigmentation pattern of $R$. solanacearum (17). Strains were stored at $-80^{\circ} \mathrm{C}$ in Difco Nutrient Broth amended with $50 \%$ glycerol.

Strain identification and genomic comparison. All $R$. solanacearum strains in this study were confirmed to species via fatty acid methyl ester (FAME) analysis. FAME analyses were performed following protocols of Miller (20) and Sasser $(28,29)$ using the MIDI Microbial Identification System and software (version TSBA 3.90; Microbial ID, Inc., Newark, DE). Biovar designations of each strain were done using procedures of Hayward (8).

Genotypic characterization. DNA was extracted from overnight broth-grown cultures using the GenomicPrep Kit (Amersham Pharmacia Biotech, Piscataway, NJ). All strains were genotyped with Rep-PCR (18). Amplifications were done using Ready-To-Go random amplified polymorphic DNA (RAPD) analysis beads (Amersham Pharmacia Biotech). Cycling parameters for the BOX-PCR were as follows: initial denaturing at $95^{\circ} \mathrm{C}$ for $5 \mathrm{~min}$ followed by 45 cycles of: $95^{\circ} \mathrm{C}$ for $1 \mathrm{~min}, 53^{\circ} \mathrm{C}$ for $1 \mathrm{~min}$, and $72^{\circ} \mathrm{C}$ for $2 \mathrm{~min}$. Profiles for ERIC and REP were generated similarly, with the exception that the annealing temperatures were adjusted to 52 and $40^{\circ} \mathrm{C}$, respectively.

After gel electrophoresis, genomic fingerprints were recorded under UV light using a Kodak Digital Documentation System 120 (Rochester, NY). Pearson correlation and unweighted pair group means analysis (UPGMA) were performed using the BioNumerics program (version 2.5; Applied Maths, Kortrijk, Belgium). For strains exhibiting unique banding patterns, DNA was extracted again, amplifications were repeated, and samples were reanalyzed. Using the BioNumerics program, a combined comparison was made of banding patterns from rep-PCR primers. Cluster cutoff values were also calculated using the BioNumerics software in each comparison.

To substantiate rep-PCR clusters of biovar 2 strains, AFLP was performed as described by Janssen et al. (14) using the EcoRI and $M s e I$ restriction enzymes, adaptors, and primers (41). Selective amplification reactions were performed using M02/E00, M03/E00, M03/E01, and M04/E01 selective nucleotide primer pairs labeled with Eco primers labeled with IR700 dye. Fragments were resolved using the Global LI-COR IR ${ }^{2}$ system. Gel images of the resulting banding patterns were recorded as Tiff files using the SAGA software (LI-COR). AFLP markers were visually confirmed and clustering analysis was done using UPGMA in BioNumerics (version 2.1; Applied Maths).

Phylotyping and sequencing of endoglucanase and the cytochrome b561 genes. Representative strains $(n=25)$ from the observed rep-PCR clusters (Fig. 1) were further analyzed. Strains were selected based on host of origin, geographic location, and the greatest difference between similarity coefficients within clusters. By doing this, we hoped to maximize genetic diversity. More strains were selected from rep-PCR clusters A, E, and F because strains within these clusters represent strains with the greatest potential of introduction and spread in North America. All procedures and primers utilized for phylotyping were those of Fegan and Prior (4). Both the endoglucanase (egl) and CybB loci were analyzed. Primers and cycling parameters for amplification and sequencing of $e g l$ have been described by Fegan et al. (5). The cytochrome $b 561$ gene was amplified using primers YP148F: 5'-CGG GCT GTT TTC TTT GGT-3' and YP148-R: 5'-CCG TCA CGC TGC TCT TG-3' or primers CYTO-F: 5'-AGG AAT CCG ATG CCG CA-3' and CYTO-R: 5'-TCA GCG CAG GAA CGG CAG CAT-3' in a reaction containing $0.4 \mu \mathrm{M}$ each primer, $0.125 \mathrm{mM}$ each dNTP, $1 \times$ standard Taq reaction buffer, and $2.5 \mathrm{U}$ of Taq DNA polymerase (New England Biolabs, Ipswich, MA). The PCR was performed on a Bio-Rad iCycler using the following program: $3 \mathrm{~min}$ at $94^{\circ} \mathrm{C}$; followed by 35 cycles of $45 \mathrm{~s}$ at $94^{\circ} \mathrm{C}, 30 \mathrm{~s}$ at 53 or $65^{\circ} \mathrm{C}$ (for CYTO-F and CYTO-R, respectively), and $45 \mathrm{~s}$ at $72^{\circ} \mathrm{C}$; and a final extension step of $10 \mathrm{~min}$ at $72^{\circ} \mathrm{C}$. YP148-F, YP148-R, CYTO-F, and CYTO-R were used for sequencing. Amplicons were visualized on agarose gels and column purified using the Wizard SV Gel and PCR Clean-up System (Promega Corp., Madison, WI). Column-purified PCR amplicons were sequenced. Independent phylogenetic analyses were conducted using the previously studied endoglucanase precursor $e g l$ and cytochrome $b-561(c y t-b)$ using the parsimony criterion implemented in PAUP $4.0 \mathrm{~b} 10$ (32). All characters were treated as unordered. Trees were constructed by a heuristic search with tree bisection-reconnection (TBR) branch swapping in a random stepwise addition of taxa repeated 1,000 times. Maxtrees was set to increase incrementally. Node support was evaluated by nonparametric bootstrap resampling (6). Bootstrap scores were calculated from 1,000 replicates, with each replicate consisting of three searches starting with a tree built by stepwise addition using the simple addition sequence. For the egl analysis, $R$. syzygii accessions AY464984 and AY465005 served as outgroup taxa. Similarly, $R$. eutropha accession AM260479 was used as an outgroup in the $c y t-b$ analysis.

Host range. The host range of race 1 strains is poorly characterized. Race 1 is known to contain strains capable of infecting a broad range of host genera and species. In contrast, race 3 bacterial wilt strains are known to have a limited host range infecting potato, tomato, geranium, and closely related solanaceous crops or weeds. A limited host range study was conducted using five R1B1 strains from each of five rep-PCR clusters B to $F$ (25 strains) and one strain of R3B2 (UW551) from cluster A. Both a stab and a nonwounding inoculation were done on three plants of the following species: tomato (cv. Walter), potato (cv. Red Pontiac), pothos (cv. Golden), tobacco (cv. Hicks), triploid banana (cv. Dwarf Cavendish), and anthurium (cv. Red Hot). For the stab inoculation, a sterile dissecting needle was dipped into a 48-h-old colony of $R$. solanacearum and stabbed into the stem of each plant $\approx 2 \mathrm{~cm}$ above the soil line. In the nonwounding experiment, 48-h-old bacteria were spectrophotometrically adjusted (absorbance at $600 \mathrm{~nm}$ ) in saline $\left(\mathrm{NaCl}\right.$ at $8.5 \mathrm{~g} /$ liter) to $1 \times 10^{8}$ $\mathrm{CFU} / \mathrm{ml}\left(6 \times 10^{7} \mathrm{CFU} / \mathrm{g}\right.$ of soil $)$ and plants were inoculated by pouring $50 \mathrm{ml}$ of the aqueous suspension onto the soil surface (9-cm pot). Plants were placed in a glasshouse with temperatures maintained at 18 to $32^{\circ} \mathrm{C}$ and maximum lighting at $266 \mu \mathrm{mol}$ $\mathrm{m}^{-2} \mathrm{~s}^{-1}$, and examined over a 6-week period for symptom development. Ralstonia spp. were reisolated, as previously described, from representative plants with wilt symptoms. Testing of the R3B2 strain was done in environmental chambers set on a 12-h day-and-night cycle $\left(19^{\circ} \mathrm{C}\right.$, night; $24^{\circ} \mathrm{C}$, day; $\left.310 \mu \mathrm{mol} \mathrm{m} \mathrm{m}^{-2} \mathrm{~s}^{-1}\right)$. In all inoculation experiments, a saline control was utilized as a noninoculated control. All host range tests were repeated under the same environmental conditions.

\section{RESULTS}

Strain identification and genomic comparison. Using FAME/MIDI analysis, strains were identified as $R$. solanacearum or closely related $R$. pickettii. However, using the FAME/MIDI analysis, strains could not be separated based on biovar, geo- 
TABLE 1. Sources of 139 Ralstonia solanacearum strains used in this study

\begin{tabular}{|c|c|c|c|c|c|c|}
\hline Strain ID (org. ID) & Source $^{\mathrm{x}}$ & Host of origin & Geographic origin ${ }^{y}$ & Origin of cuttings & Year & Biovar \\
\hline P446 & 5 & Pothos & Florida & Costa Ricaz & 1996 & 1 \\
\hline P447 & 5 & Pothos & Florida & Costa Rica & 1996 & 1 \\
\hline P448 & 5 & Pothos & Florida & Costa Rica & 1996 & 1 \\
\hline P449 & 5 & Pothos & Florida & Costa Rica & 1996 & 1 \\
\hline P485 & 5 & Pothos & Florida & Costa Rica & 1996 & 1 \\
\hline P486 & 5 & Pothos & Florida & Costa Rica & 1996 & 1 \\
\hline P487 & 5 & Pothos & Florida & Costa Rica & 1996 & 1 \\
\hline P488 & 5 & Pothos & Florida & Costa Rica & 1996 & 1 \\
\hline P489 & 5 & Pothos & Florida & Costa Rica & 1996 & 1 \\
\hline P490 & 5 & Pothos & Florida & Costa Rica & 1996 & 1 \\
\hline P491 & 5 & Pothos & Florida & Costa Rica & 1996 & 1 \\
\hline P492 & 5 & Pothos & Florida & Costa Rica & 1996 & 1 \\
\hline P493 & 5 & Pothos & Florida & Costa Rica & 1996 & 1 \\
\hline P494 & 5 & Pothos & Florida & Costa Rica & 1996 & 1 \\
\hline P495 & 5 & Pothos & Florida & Costa Rica & 1996 & 1 \\
\hline P496 & 5 & Pothos & Florida & Costa Rica & 1996 & 1 \\
\hline P497 & 5 & Pothos & Florida & Costa Rica & 1996 & 1 \\
\hline P498 & 5 & Pothos & Florida & Costa Rica & 1996 & 1 \\
\hline P499 & 5 & Pothos & Florida & Costa Rica & 1996 & 1 \\
\hline P500 & 5 & Pothos & Florida & Costa Rica & 1996 & 1 \\
\hline P501 & 5 & Pothos & Florida & Costa Rica & 1996 & 1 \\
\hline P502 & 5 & Pothos & Florida & Costa Rica & 1996 & 1 \\
\hline P503 (PS 1425 VI) & 7 & Tomato & Florida & $\ldots$ & 1996 & 1 \\
\hline P504 (PS 1525 VI) & 7 & Tomato & Florida & $\ldots$ & 1996 & 1 \\
\hline P506 & 5 & Pothos & Florida & Costa Rica & 1996 & 1 \\
\hline P507 & 5 & Pothos & Florida & Costa Rica & 1996 & 1 \\
\hline P508 & 5 & Pothos & Florida & Costa Rica & 1996 & 1 \\
\hline P509 & 5 & Pothos & Florida & Costa Rica & 1996 & 1 \\
\hline P514 & 5 & Pothos & Florida & Costa Rica & 1996 & 1 \\
\hline P515 & 5 & Pothos & Florida & Costa Rica & 1996 & 1 \\
\hline P517 & 5 & Pothos & Florida & Costa Rica & 1996 & 1 \\
\hline P521 & 5 & Pothos & Florida & Costa Rica & 1996 & 1 \\
\hline P522 & 5 & Pothos & Florida & Costa Rica & 1996 & 1 \\
\hline P523 & 5 & Pothos & Florida & Costa Rica & 1996 & 1 \\
\hline P524 & 5 & Pothos & Florida & Costa Rica & 1996 & 1 \\
\hline P525 & 5 & Pothos & Florida & Costa Rica & 1996 & 1 \\
\hline P526 & 5 & Pothos & Florida & Costa Rica & 1996 & 1 \\
\hline P527 & 5 & Pothos & Florida & Costa Rica & 1996 & 1 \\
\hline P528 (ATCC 33192) & 1 & Physalis angulata & Florida & $\ldots$ & $\ldots$ & 1 \\
\hline P529 (ATCC 33193) & 1 & Potato & Florida & $\ldots$ & $\ldots$ & 3 \\
\hline P530 (1541-1) & 4 & Tomato & Florida & $\ldots$ & 1996 & 1 \\
\hline P531 (1541-5) & 4 & Tomato & Florida & $\ldots$ & 1996 & 1 \\
\hline P532 & 6 & Tomato & Florida & $\ldots$ & 1996 & 1 \\
\hline P533 (H4) & 6 & Tomato & Florida & $\ldots$ & 1996 & 1 \\
\hline P534 (6) & 6 & Tomato & Florida & $\ldots$ & 1996 & 1 \\
\hline P535 (8) & 6 & Tomato & Florida & $\ldots$ & 1996 & 1 \\
\hline P536 (10) & 6 & Tomato & Florida & $\ldots$ & 1996 & 1 \\
\hline P541 (T5) & 8 & Potato & Florida & $\ldots$ & 1996 & 1 \\
\hline P543 & 8 & Potato & Florida & $\ldots$ & 1994 & 1 \\
\hline P545 & 5 & Pothos & Florida & Costa Rica & 1997 & 1 \\
\hline P546 & 5 & Pothos & Florida & Costa Rica & 1997 & 1 \\
\hline P547 & 5 & Pothos & Florida & Costa Rica & 1997 & 1 \\
\hline P548 & 5 & Pothos & Florida & Costa Rica & 1997 & 1 \\
\hline P549 & 8 & Potato & Florida & $\ldots$ & 1997 & 1 \\
\hline P550 & 8 & Potato & Florida & $\ldots$ & 1997 & 1 \\
\hline P551 & 8 & Potato & Florida & $\ldots$ & 1997 & 1 \\
\hline P552 & 8 & Potato & Florida & $\ldots$ & 1997 & 1 \\
\hline P553 & 8 & Potato & Florida & $\ldots$ & 1997 & 1 \\
\hline P557 & 5 & Anthurium & Florida & Costa Rica & 1997 & 1 \\
\hline P558 & 5 & Anthurium & Florida & Costa Rica & 1997 & 1 \\
\hline
\end{tabular}

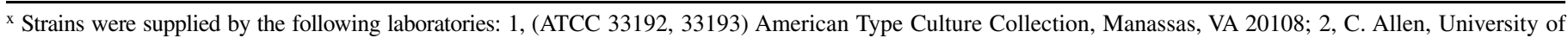
Wisconsin-Madison, Madison, WI 53706; 3, (BCCM/LMG 2294, 2298, 2300, 2306, 17139, 17140, 17141, 17142) Belgian Coordinated Collections of Microorganisms, Brussels; 4, Division of Plant Industry, PO Box 147100 Gainesville, FL 32614; 5, Mid-Florida Research and Education Center, University of Florida, Apopka 32703; 6, D. Chellemi, North-Florida Research and Education Center, University of Florida, Quincy 32351; 7, J. Jones, Gulf Coast Research and Education Center, University of Florida, Bradenton 34945; 8, D. P. Weingartner, Hasting Research and Education Center, University of Florida, Hastings 32145; 9,. P. Prior, INRA, Station of Vegetable Pathology, Cedex, France; 10, P. Roger, Centre for plant Quarantine Pests, Canada Food Inspection Agency, Ontario, Canada; 11, M. Zapata, Crop Protection Department, University of Puerto Rico, P.O. Box 9030, Mayaguez, Puerto Rico 00681-9030; 12, M. Daughtrey, Department of Plant Pathology, Cornell University, 3059 Sound Ave., Riverhead, NY 11901; 13, P. Roberts, Southwest Florida Research and Education Center, University of Florida, 2686 SR 29 North, Immokalee 34142; 14, A. Darrasse, INRA URPV, Domaine Duclos, F-97170 Petit-Bourg; 15, D. Thomas, Goldsmith Inc., P.O. Box 1349, Gilroy, CA 95021.

y Geographic origin of United States used when exact state is not known.

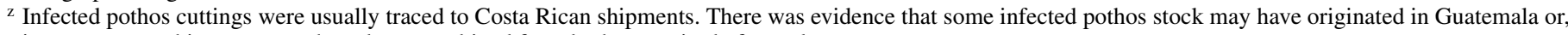
in some cases, shipments may have been combined from both countries before sale. 
TABLE 1. (continued from preceding page)

\begin{tabular}{|c|c|c|c|c|c|c|}
\hline Strain ID (org. ID) & Source $^{\mathrm{a}}$ & Host of origin & Geographic origin ${ }^{b}$ & Origin of cuttings & Year & Biovar \\
\hline P559 & 5 & Anthurium & Florida & Costa Rica & 1997 & 1 \\
\hline P565 & 5 & Pothos & Florida & Costa Rica & 1998 & 1 \\
\hline P574 & 5 & Pothos & Florida & Costa Rica & 1999 & 1 \\
\hline P575 & 5 & Pothos & Florida & Costa Rica & 1999 & 1 \\
\hline P576 & 13 & Tomato & Florida & $\ldots$ & 1999 & 1 \\
\hline P577 & 13 & Tomato & Florida & $\ldots$ & 1999 & 1 \\
\hline P578 (12-4-1) & 12 & Geranium & United States & Guatemala & 1999 & 2 \\
\hline P579 (12-4-3) & 12 & Geranium & United States & Guatemala & 1999 & 2 \\
\hline P580 (12-4-5) & 12 & Geranium & United States & Guatemala & 1999 & 2 \\
\hline P581 (12-4-6) & 12 & Geranium & United States & Guatemala & 1999 & 2 \\
\hline P582 (1135-11) & 12 & Geranium & Florida & Guatemala & 1999 & 2 \\
\hline P583 (1135-12) & 12 & Geranium & Florida & Guatemala & 1999 & 2 \\
\hline P589 & 13 & Tomato & Florida & $\ldots$ & 1999 & 1 \\
\hline P594 & 5 & Tomato & Florida & $\ldots$ & 1999 & 1 \\
\hline P596 & 5 & Tomato & Florida & $\ldots$ & 1999 & 1 \\
\hline P597 & 5 & Tomato & Florida & $\ldots$ & 1999 & 1 \\
\hline P598 & 5 & Tomato & Florida & $\ldots$ & 1999 & 1 \\
\hline P606 (8217) & 14 & Tomato & Guadeloupe & $\ldots$ & 1985 & 1 \\
\hline P607 (a12) & 14 & Tomato & Guadeloupe & $\ldots$ & 1993 & 1 \\
\hline P608 (GT4) & 14 & Tomato & Guadeloupe & $\ldots$ & 1984 & 3 \\
\hline P609 (MT1) & 14 & Tomato & Martinique & $\ldots$ & 1986 & 3 \\
\hline P610 (MT5) & 14 & Tomato & Martinique & $\ldots$ & 1987 & 1 \\
\hline P611 (G34) & 14 & Potato & Guadeloupe & $\ldots$ & 1993 & 3 \\
\hline P612 (82GPT117) & 14 & Potato & Guadeloupe & $\ldots$ & 1987 & 1 \\
\hline P613 (MPT1) & 14 & Potato & Martinique & $\ldots$ & 1986 & 1 \\
\hline P614 (B62) & 14 & Eggplant & Guadeloupe & $\ldots$ & 1993 & 1 \\
\hline P615 (GA3) & 14 & Eggplant & Guadeloupe & $\ldots$ & 1984 & 3 \\
\hline P617 (99.307) & 9 & Anthurium & Martinique & $\ldots$ & 1999 & 1 \\
\hline P618 (99.1119/1) & 9 & Anthurium & Martinique & $\ldots$ & 1999 & 1 \\
\hline P619 (99.1119/2) & 9 & Anthurium & Martinique & $\ldots$ & 1999 & 1 \\
\hline P620 (99.1120/1) & 9 & Anthurium & Martinique & $\ldots$ & 1999 & 1 \\
\hline P621 (99.1120/2) & 9 & Anthurium & Martinique & $\ldots$ & 1999 & 1 \\
\hline P622 (99.1121/1) & 9 & Anthurium & Martinique & $\ldots$ & 1999 & 1 \\
\hline P623 (99.1121/2) & 9 & Anthurium & Martinique & $\ldots$ & 1999 & 1 \\
\hline P624 (99.1122) & 9 & Anthurium & Martinique & $\ldots$ & 1999 & 1 \\
\hline P625 (99.1542) & 9 & Anthurium & Martinique & $\ldots$ & 1999 & 1 \\
\hline P626 (00.116) & 9 & Anthurium & Martinique & $\ldots$ & 2000 & 1 \\
\hline P627 & 15 & Geranium & United States & Guatemala & 2000 & 2 \\
\hline P629 & 15 & Geranium & United States & Guatemala & 2000 & 2 \\
\hline P630 & 15 & Geranium & United States & Guatemala & 2000 & 2 \\
\hline P631 & 15 & Geranium & United States & Guatemala & 2000 & 2 \\
\hline P632 & 15 & Geranium & United States & Guatemala & 2000 & 2 \\
\hline P640 & 15 & Geranium & United States & Guatemala & 2001 & 2 \\
\hline P641 & 15 & Geranium & United States & Guatemala & 2001 & 2 \\
\hline P651 (1977) & 11 & Heliconia & Puerto Rico & $\ldots$ & 2001 & 1 \\
\hline P654 (1980) & 11 & Heliconia & Puerto Rico & $\ldots$ & 2001 & 1 \\
\hline P658 (LMG 2294) & 3 & Potato & Columbia & $\ldots$ & $\ldots$ & 2 \\
\hline P660 (LMG 2299) & 3 & Tomato & USA & $\ldots$ & 1953 & 1 \\
\hline P661 (LMG 2300) & 3 & Potato & Israel & $\ldots$ & 1954 & 2 \\
\hline P662 (LMG 2306) & 3 & Tomato & Portugal & $\ldots$ & 1960 & 2 \\
\hline P664 (LMG 9673) & 3 & Geranium & Reunion Island & $\ldots$ & 1961 & 1 \\
\hline P665 (LMG 17139) & 3 & Potato & Cyprus & $\ldots$ & 1963 & 2 \\
\hline P666 (LMG 17140) & 3 & Potato & Sweden & $\ldots$ & 1972 & 2 \\
\hline P667 (LMG 17141) & 3 & Potato & $\ldots$ & $\ldots$ & 1982 & 2 \\
\hline P668 (LMG 17142) & 3 & Potato & England & $\ldots$ & 1996 & 2 \\
\hline P673 & 5 & Pothos & Florida & $\ldots$ & 2003 & 1 \\
\hline P674 & 15 & Geranium & United States & Kenya & 2003 & 2 \\
\hline P675 & 15 & Geranium & United States & Kenya & 2003 & 2 \\
\hline P676 & 15 & Geranium & United States & Kenya & 2003 & 2 \\
\hline P677 & 15 & Geranium & United States & Kenya & 2003 & 2 \\
\hline P678 & 15 & Geranium & United States & Kenya & 2003 & 2 \\
\hline P680 & 15 & Geranium & United States & Kenya & 2003 & 2 \\
\hline P694 (B03162) & 10 & Geranium & Canada & Costa Rica & 2003 & 2 \\
\hline P695 (B03163) & 10 & Geranium & Canada & Costa Rica & 2003 & 2 \\
\hline P696 (B03164) & 10 & Geranium & Canada & Costa Rica & 2003 & 2 \\
\hline P697 (B03165) & 10 & Geranium & Canada & Costa Rica & 2003 & 2 \\
\hline P698 (B03166) & 10 & Geranium & Canada & Costa Rica & 2003 & 2 \\
\hline P699 (B03167) & 10 & Geranium & Canada & Costa Rica & 2003 & 2 \\
\hline P700 (B03168) & 10 & Geranium & Canada & Costa Rica & 2003 & 2 \\
\hline P701 (B03169) & 10 & Geranium & Canada & Costa Rica & 2003 & 2 \\
\hline P702 (B03170) & 10 & Geranium & Canada & Costa Rica & 2003 & 2 \\
\hline P703 (B03171) & 10 & Geranium & Canada & Costa Rica & 2003 & 2 \\
\hline P704 (B03173) & 10 & Geranium & Canada & Costa Rica & 2003 & 2 \\
\hline
\end{tabular}


graphic origin, or original host. rep-PCR was effective in separating strains into distinct clusters based on origin and biovar (Fig. 1). Biovar 1 strains were separated into five clusters. Similarity coefficients in clusters were $\geq 70 \%$ except for cluster D. This cluster contained strains isolated from vegetables and heliconia and appears to be very genotypically diverse in nature. Biovar 2 strains were separated into one cluster with four subclusters. In confirming validity of these four subclusters, DNA was extracted at least twice from each strain and PCR was performed with the rep-primers a minimum of two times. In these repeated analyses, strains recovered from Guatemala could not always be separated from world-wide control strains, nor could the Kenya strains always be separated from Costa Rican strains. It appears that faint bands in combination with computer-assisted strain separation may be responsible for inability to consistently differentiate populations. The Guatemala strains were isolated over a 4-year period (1999-2002) and appear to exhibit moderate genotypic diversity. The strains from Kenya formed a tight cluster and likely represent a clonal population. These strains were recovered in 2002 from geraniums imported from Kenya. The R3B2 strains of Costa Rican origin were also isolated in 2002; however, from cuttings imported into Canada. In order to confirm subclusters in biovar 2, four different primer pairs were employed in AFLP analysis. Using AFLP, it was not possible to distinguish populations regardless of the number of AFLP markers used. However, unique polymorphic fragments were observed for some isolates that might prove useful in distinguishing biovar 2 populations. Because these rep-PCR subclusters could not reliably be reproduced, separation is not shown in Figure 1.

Phylotyping and sequencing of endoglucanase and the cytochrome b561 genes. Using multiplex PCR, the majority of strains were classified as phylotype II, with only two reference strains of race 1 , biovar 3 being phylotype 1 (Fig. 2). Biovar 3 strains in phylotype I are rarely found in the United States. Phylotype II comprises strains belonging to biovars 1 and 2 isolated in America and includes races 2 and 3 , and some race 1 strains $(3,4)$. The $e g l$ gene sequence has been used successfully to distinguish $R$. solanacearum into 23 sequevars (4). In our analysis, three well-supported separate clades were observed within phylotype II (Fig. 2). All (R3B2) strains from rep-PCR cluster A were found to have one $e g l$ sequence type (sequevar 1). Similarly, the R1B1 strains from either pothos or anthurium (rep-PCR clusters $\mathrm{B}, \mathrm{E}$, and $\mathrm{F}$ ) shared one egl sequence type (sequevar 4). In contrast, R1B1 strains recovered from vegetable hosts (rep-PCR clusters $\mathrm{C}$ and $\mathrm{D})$ exhibit more diversity in the egl region (sequevar 5 and 7). Similar observations using the $e g l$ locus have been previously reported by Castillo and Greenberg (3). In this study, both the egl and cytochrome $b 561$ sequences allowed for similar genotypic resolution of $R$. solanacearum strains (Figs. 2 and 3).

rep-PCR can be used effectively to distinguish clonal outbreaks (lines) of bacterial wilt. rep-PCR fingerprinting cannot effectively be used to distinguish populations into large infrasubspecific groups (sequevars).

Host range. Strains in different rep-PCR clusters exhibited different host ranges (Table 2). The larger host ranges were observed with strains in rep-PCR clusters B, E, and F (sequevar 4) representing strains from Costa Rica, Martinique, and Guadeloupe. These strains were isolated from anthurium and pothos and were virulent on tomato, potato, and geranium, infecting and causing wilt using either wounding or soil drench inoculation methods. Strains in clusters $\mathrm{E}$ and $\mathrm{F}$ also produced wilt symptoms on anthurium and pothos using either inoculation method. Strains in cluster B only produced wilt symptoms on pothos using the wound inoculation method and did not wilt tobacco. The race 1 strains, isolated from vegetables, in clusters C and D (sequevars 5 and 7) produced wilt symptoms on tomato, potato, and geranium using either inoculation method. Only $40 \%$ of the strains in these two clusters produced wilt symptoms on tobacco. As expected, the R3B2 (UW551) strain (sequevar 1) only infected tomato, potato, and geranium. Geranium may be a universal host of biovar 1 and 2, similar to tomato and potato. Undoubtedly, we would expect variation in susceptibility of geranium cultivars as seen in cultivars of tomato and potato.

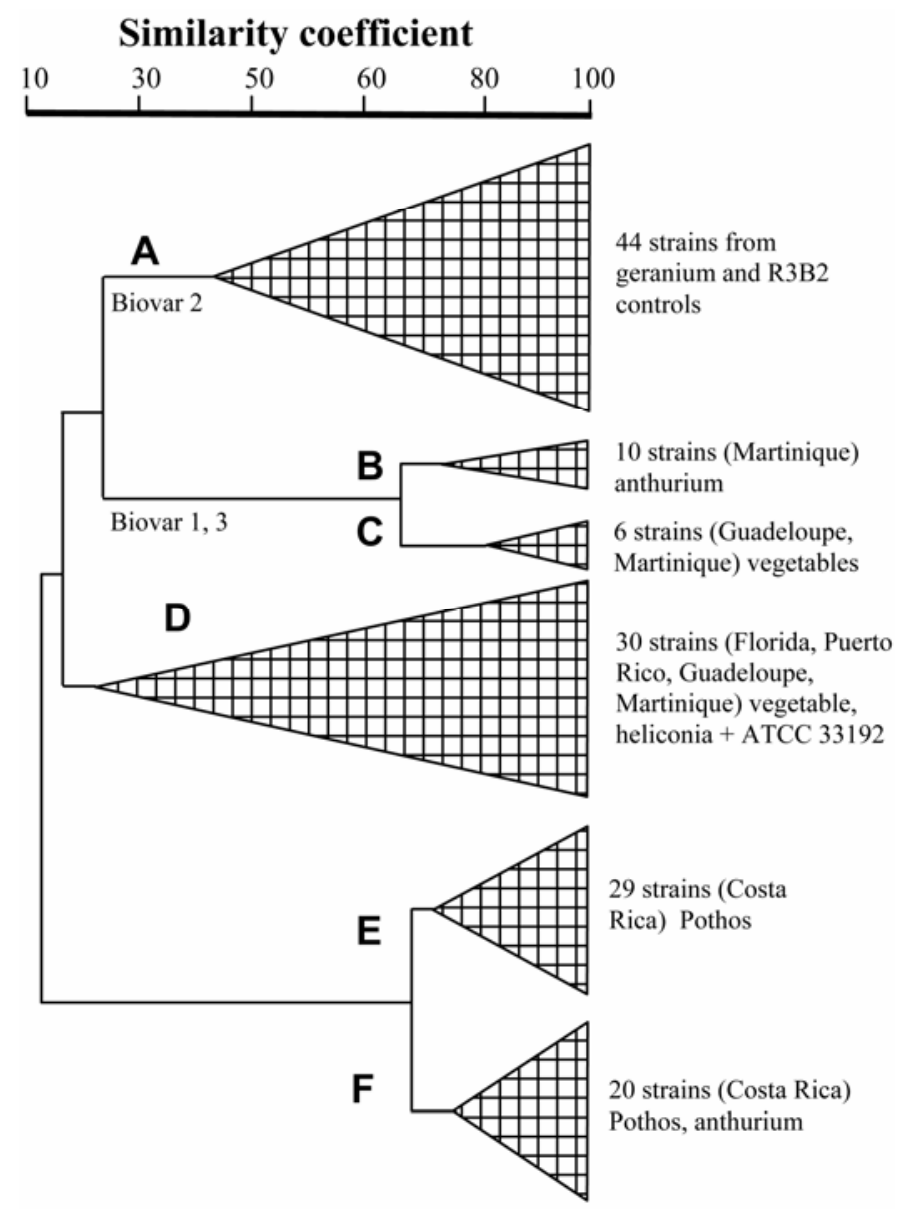

Fig. 1. Cluster analysis generated by BioNumerics program (version 2.5) using combined profiles of 139 Ralstonia solanacearum strains generated by repetitive polymerase chain reaction primer sets (BOX, ERIC, and REP). Cluster A contained all R3B2 strains. The other five clusters contained strains of biovar 1 and 3 from the United States, Central America, and the Caribbean basin.

TABLE 1. (continued from preceding page)

\begin{tabular}{|c|c|c|c|c|c|c|}
\hline Strain ID (org. ID) & Source $^{\mathrm{a}}$ & Host of origin & Geographic origin ${ }^{\mathrm{b}}$ & Origin of cuttings & Year & Biovar \\
\hline P705 (B03174) & 10 & Geranium & Canada & Costa Rica & 2003 & 2 \\
\hline P706 (B03194) & 10 & Geranium & Canada & Costa Rica & 2003 & 2 \\
\hline P707 (B03196) & 10 & Geranium & Canada & Costa Rica & 2003 & 2 \\
\hline P709 (B03200) & 10 & Geranium & Canada & Costa Rica & 2003 & 2 \\
\hline P714a (UW551) & 2 & Geranium & Wisconsin & Kenya & 2003 & 2 \\
\hline
\end{tabular}




\section{DISCUSSION}

Many ornamental crops are produced in countries that have low labor costs as well as environments favorable to plant growth. Each year, thousands of ornamental plants are brought into the United States. The majority of these arrive as either cuttings, bare root, or some other type of asexual propagule. The sheer volume makes it impossible to accurately check for systemic pathogens. Therefore, inspection emphasis is placed on insects and fungal pathogens that produce spore structures.

In order to safeguard the geranium industry, strict production guidelines have been implemented for facilities producing geranium cuttings that are shipped into the United States (37). Sanitation measures include raised benches, cement floors, purified water, sterilization of tools, and the use of synthetic growing media. Before these sanitation measures were implemented, imported geraniums from Africa and Central America were occasionally found to be infected with R3B2. Geraniums have also been linked to introductions of R3B2 into Europe (13). A reasonable concern has always been that $R$. solanacearum would be introduced and spread to potato production. However, to date, there has been no evidence of spread from geranium production to potato. Potato cultivars are highly susceptible to these introduced strains; however, without cocultivation, establishment in potato-producing areas is unlikely. In order to establish $R$. solanacearum in a temperate environment, a host must be readily available and there must be a mechanism of movement (i.e., water) (26).

Because of the international movement of ornamental plants, it is important to be able to determine the origin of bacterial wilt outbreaks. A quick, accurate method of distinguishing new populations of the pathogen is required. Common diagnostic methods such as BIOLOG (1) and FAME analysis (12) have been advocated in studying bacterial wilt populations. However, these techniques require an extended time period and specialized equipment. In addition, library systems that are sold for identification commonly misidentify strains due to the large genetic diversity found within $R$. solanacearum. In this study, FAME/ MIDI analysis was usually accurate in identifying Ralstonia spp. but could not separate strains based on race, biovar, original host, or country of origin.

In recent years, the $16 \mathrm{~S}$ rDNA $(11,25,33)$, egl (4), and hrpB (24) genes have been used to separate strains of $R$. solanacearum into genetic divisions that can be traced back to continent of origin (40). The various levels of polymorphism that exist within each of these DNA sequences determine their usefulness. Genomic fingerprinting using RAPD, AFLP, pulsed-field gel electrophoresis, and rep-PCR have been recommended in studies that are trying to examine clonal lines (4). A number of studies have used rep-PCR to distinguish populations of $R$. solanacearum $(10,15,27,35)$. In this study, rep-PCR was useful in separating strains based on biovar and, to an extent, host and country of origin. Being able to identify clonal lines is very useful because most outbreaks within production facilities are considered to arise from a single introduced clone of the pathogen. This is due to asexual crop propagation and on-site spread of the pathogen by workers within a production facility. The highest level of resolution observed with rep-PCR was obtained when data from all three primer sets were analyzed in combination. Intensity of various gels was equalized using a standard molecular weight ladder. Except for removal of occasional dirt spots and faint bands below $298 \mathrm{bp}$, scoring of rep-PCR-generated markers required no editing. Scoring markers manually by selecting only those with greater intensity did not allow for any greater resolution in these populations.

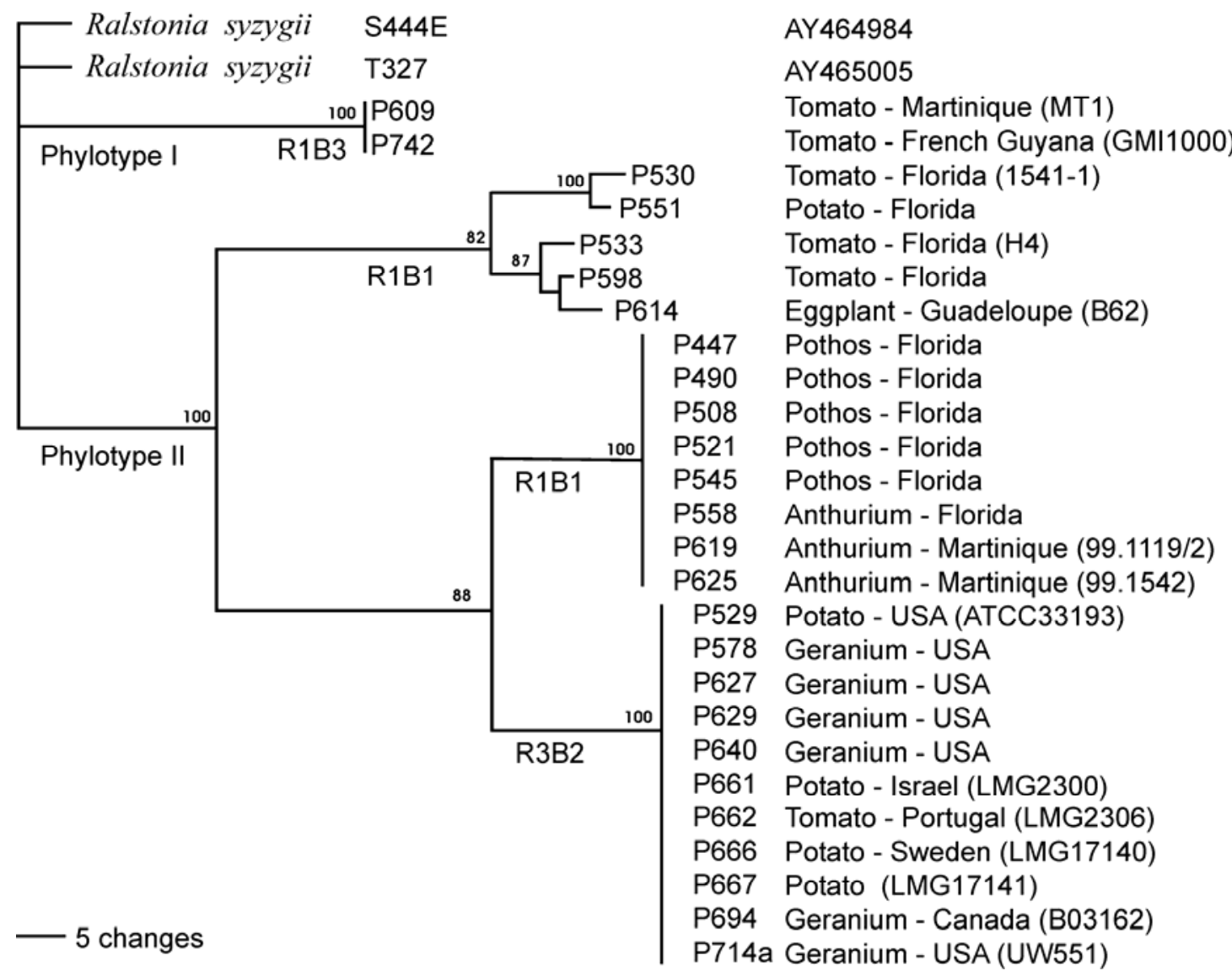

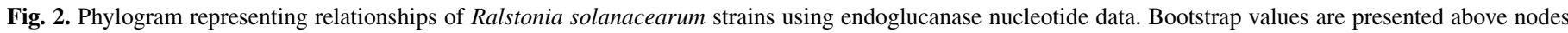

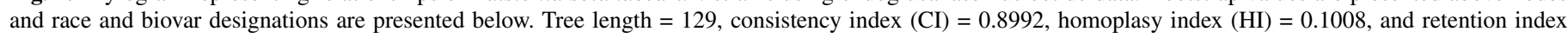
$(\mathrm{RI})=0.967$. 
Using rep-PCR, biovar 3 could easily be distinguished from biovars 1 and 2 . However, within cluster A, subclusters were evident only in certain analysis runs and could not always be distinguished with rep-PCR. The lower limit of utility of rep-PCR was observed when trying to distinguish clonal populations of R3B2. The strains originating from Kenya always appeared to be distinct and probably clonal in nature. Likewise, in this study, AFLP markers were not particularly useful in distinguishing R3B2 populations to the level of geographical origin level. The lowest rep-PCR similarity coefficients observed in this study were in cluster $\mathrm{D}$, suggesting that biovar 1 strains isolated from vegetables in Florida and the Caribbean islands are perhaps under less selection when compared with the other races. In regards to the biovar 1 strains, our results are in agreement with previous research revealing a high degree of genetic heterogeneity found in the United States $(19,39)$.

The sequevar classification system based on the egl gene sequence has been very useful in identifying infrasubspecific groups of $R$. solanacearum (4). The low similarity coefficients of the repPCR clusters compared with the sequevar clusters substantiates that there are many clonal lines making up each sequevar cluster. Similarity coefficients and strains within each cluster were nearly identical when either the $e g l$ or cytochrome $b 561$ gene sequence was compared (Fig. 2). It appears that either sequence could be used to distinguish $R$. solanacearum sequevars. Because rep-PCR produces a specific genomic fingerprint for a clonal line, it cannot effectively be used to distinguish the larger infrasubspecific groups (sequevars).

$R$. solanacearum is known to infect hundreds of plant species (9). Biovar 1 strains are known to have the largest host range, although individuals within this group can have very limited host ranges. Comparatively, biovar 2 strains are known to have limited host ranges, primarily infecting solanaceous crops and weeds (13). Still other plant species can act as latent hosts in which bacteria colonize both the vascular systems and roots without symptom development (13). The limited host range tested in this
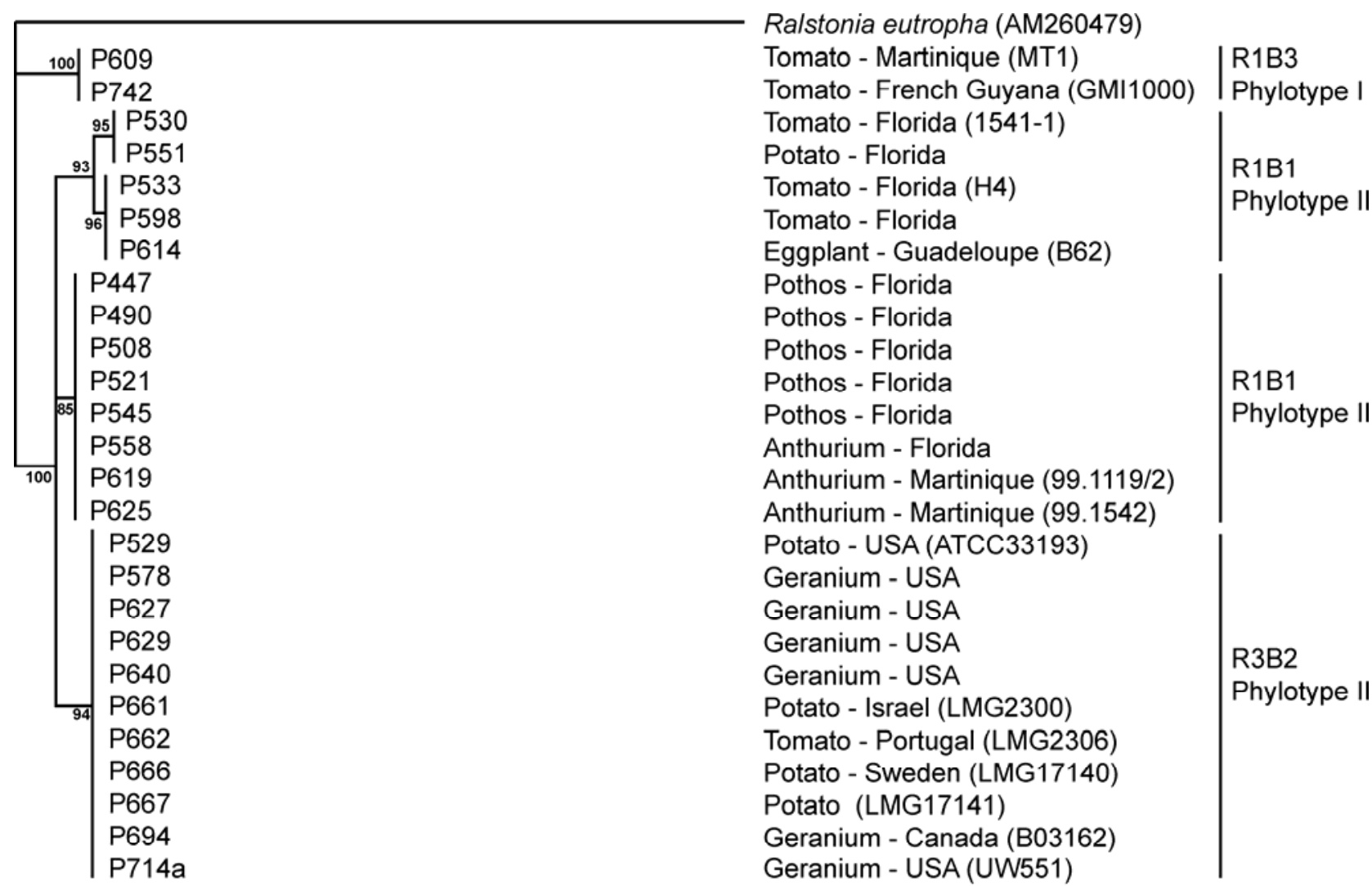

Fig. 3. Phylogram representing relationships of Ralstonia solanacearum strains using cytochrome $b-561$ nucleotide data. Bootstrap values are presented above nodes and race and biovar designations are presented on vertical bars to the right. Tree length $=166$, consistency index $(\mathrm{CI})=0.9518$, homoplasy index $(\mathrm{HI})=$ $0.0482, \mathrm{CI}$, and retention index $(\mathrm{RI})=0.9477$.

TABLE 2. Combined host range data from both the wound and drench inoculations ${ }^{\vee}$

\begin{tabular}{|c|c|c|c|c|c|c|c|c|}
\hline Rep-PCR cluster & Sequevar & Tobacco & Tomato & Potato & Banana & Pothos & Anthurium & Geranium \\
\hline A & 1 & $-\mathrm{w}$ & $t^{\mathrm{x}}$ & $t^{x}$ & $-\mathrm{w}$ & $-^{\mathrm{w}}$ & $-\mathrm{w}$ & $t^{\mathrm{x}}$ \\
\hline $\mathrm{C}$ & 5 & $t^{\mathrm{x}}$ & $t^{\mathrm{x}}$ & $t^{\mathrm{x}}$ & -w $^{\mathrm{w}}$ & -w $^{\mathrm{w}}$ & ${ }^{\mathrm{w}}$ & $t^{\mathrm{x}}$ \\
\hline $\mathrm{F}$ & 4 & $t^{x}$ & $t^{\mathrm{x}}$ & $+^{\mathrm{x}}$ & $-\mathrm{y}$ & $+^{x}$ & $+^{\mathrm{x}}$ & $t^{\mathrm{x}}$ \\
\hline
\end{tabular}

${ }^{v}$ Five strains from each repetitive polymerase chain reaction (rep-PCR) cluster were tested, except for cluster A, from which only one strain was used (UW551).

${ }^{w}$ No wilt symptoms observed with either inoculation procedure.

${ }^{x}$ Wilt symptoms produced with both wound and drench inoculations.

y Vascular discoloration occurred; however, wilt or death of plants rarely occurred.

z Wilt symptoms only observed with wound inoculations. 
study illustrates that the strains arriving in North America are poorly characterized. Hosts which they are capable of infecting are unknown and their ability to become established is equally unknown.

\section{ACKNOWLEDGMENTS}

This research was funded by the United States Department of Agriculture Floral Industry Task Force Specific Cooperative Agreement, The Fred C. Gloeckner Foundation, and the University of Florida Institute of Food and Agricultural Sciences.

\section{LITERATURE CITED}

1. Black, R., and Sweetmore, A. 1992. Identification and characterization of Pseudomonas solanacearum using metabolic profiles. In: Bacterial Wilt Proceedings of an International Symposium, Kaohsiung, Taiwan, ROC. G. L. Hartman and A. C. Hayward, eds. ACIAR Proc. 45:32-44.

2. Buddenhagen, I. W. 1961. Bacterial wilt of bananas: history and known distribution. Trop. Agric. (Trinidad) 38:107-121.

3. Castillo, J. A., and Greenberg, J. T 2007. Evolutionary dynamics of Ralstonia solanacearum. Appl. Environ. Microbiol. 73:1225-1238.

4. Fegan, M., and Prior, P. 2005. How complex is the Ralstonia solanacearum species complex? Pages 449-461 in: Bacterial Wilt: The Disease and the Ralstonia solanacearum Species Complex. C. Allen, P. Prior, and A. C. Hayward, eds. American Phytopathological Society, St. Paul, MN.

5. Fegan, M., and Prior, P. 2006. Diverse members of the Ralstonia solanacearum species complex cause bacterial wilts of banana. Aust. Plant Pathol. 35:93-101.

6. Felsenstein, J. 1985. Confidence limits on phylogenies: an approach using the bootstrap. Evolution 39:783-791.

7. Harrison, D. E. 1961. Bacterial wilt of potatoes, field symptoms of the disease and studies on the causal organism, Pseudomonas solanacearum variety asiaticum. Aust. J. Agric. Res. 12:854-871.

8. Hayward, A. C. 1964. Characteristics of Pseudomonas solanacearum. J. Appl. Bacteriol. 27:265-277.

9. Hayward, A. C. 1991. Biology and epidemiology of bacterial wilt caused by Pseudomonas solanacearum. Annu. Rev. Phytopathol. 29:65-87.

10. Horita, M., and Tsuchiya, K. 2000. Comparative analysis of Japanese and foreign strains of Ralstonia solanacearum based on 16S ribosomal RNA gene sequences. J. Gen. Plant Pathol. 66:132-137.

11. Horita, M., and Tsuchiya, K. 2000. Genetic diversity of Japanese strains of Ralstonia solanacearum. Phytopathology 91:399-407.

12. Janse, J. D. 1991. Infra- and intraspecific classification of Pseudomonas solanacearum strains, using whole cell fatty acid analysis. Syst. Appl. Microbiol. 14:335-345.

13. Janse, J. D., van den Beld, H. E., Elphinstone, J., Simpkins, S., Tjou-TamSin, N. A. A., and van Vaerenbergh, J. 2004. Introduction to Europe of Ralstonia solanacearum biovar 2, race 3 in Pelargonium zonale cuttings. J. Plant Pathol. 86:147-155.

14. Janssen, P., Coopman, R., Huys, G., Swings, J., Bleeker, M., Vos, P., Zabeau, M., and Kersters, K. 1996. Evaluation of the DNA fingerprinting method AFLP as a new tool in bacterial taxonomy. Microbiology 142:1881-1893.

15. Jaunet, T. X., and Wang, J. F. 1999. Variation in genotype and aggressiveness diversity of Ralstonia solanacearum race 1 isolated from tomato in Taiwan. Phytopathology 89:320-327.

16. Jones, R. K. 1993. Southern Bacterial wilt. Pages 242-245 in: Geraniums IV. J. White, ed. J. W. Ball Publishing, Geneva, IL.

17. Kelman, A. 1954. The relationship of pathogenicity in Pseudomonas solanacearum to colony appearance on tetrazolium medium. Phytopathology 44:693-695.

18. Louws, F. J., Fulbright, D. W., Stephens, C. T., and de Bruijn, F. J. 1994 Specific genomic fingerprints of phytopathogenic Xanthomonas and Pseudomonas pathovars and strains generated with repetitive sequences and PCR. Appl. Environ. Microbiol. 60:2286-2295.

19. McLaughlin, R. J., and Sequeira, L. 1989. Phenotypic diversity in strains of Pseudomonas solanacearum isolated from a single potato field in Northeastern Florida. Plant Dis. 73:960-964.

20. Miller L. T. 1982. Single derivation method for routine analysis of bacterial whole-cell fatty acid methyl esters, including hydroxy acids. J. Clin. Microbiol. 16:584-586.

21. Norman, D., and Alvarez, A. 1989. A rapid method for the presumptive identification of Xanthomonas campestris pv. dieffenbachiae and other xanthomonads. Plant Dis. 73:654-658.

22. Norman, D. J., and Yuen, J. M. F. 1998. A distinct pathotype of Ralstonia (Pseudomonas) solanacearum race 1, biovar 1 entering Florida on infected pothos (Epipremnum aureum) cuttings. Can. J. Plant Pathol. 20:171-175.

23. Norman, D. J., and Yuen, J. M. F. 1999. First report of Ralstonia (Pseudomonas) solanacearum infecting pot anthurium production in Florida. Plant Dis. 83:300.

24. Poussier, S., Prior, P., Luisetti, J., Hayward, C., and Fegen, M. 2000 Partial sequencing of the $h r p \mathrm{~B}$ and endoglucanase genes confirms and expands the know diversity within the Ralstonia solanacearum species complex. Syst. Appl. Microbiol. 23:479-486.

25. Poussier, S., Trigalet-Demery, D., Vandewalle, P., Goffinet, B., Luisetti, J., and Trigalet, A. 2000. Genetic diversity of Ralstonia solanacearum as assessed by PCR-RFLP of the hrp gene region, AFLP, and 16S rRNA sequence analysis and identification of an African subdivision. Microbiology 146:1679-1692.

26. Rafoss, T. 2003. Spatial stochastic simulation offers potential as a quantitative method for pest risk analysis. Risk Anal. 23:651-661.

27. Robertson, A. E., Fortnum, B. A., Wood, T. C., and Kluepfel, D.A. 2001. Diversity of Ralstonia solanacearum in the Southern United States. Contrib. Tob. Res. 19:323-331.

28. Sasser, M. J. 1990. Identification of bacteria through fatty acid analysis. Pages 199-204 in: Methods in Phytobacteriology. Z. Klement, K. Rudolph, and D. Sands, eds. Akademiai Kiado, Budapest.

29. Sasser, M. J. 1990. Technical note \#101: identification of bacteria by gas chromatography of cellular fatty acids. MIDI, 115 Barksdale Professional Center, Newark, DE.

30. Strider, D. L., Jones, R. K., and Haygood, R. A. 1981. Southern bacterial wilt of geranium caused by Pseudomonas solanacearum. Plant Dis. 65:52-53.

31. Swanson, J. K., Yao, J., Tans-Kersten, J., and Allen, C. 2005. Behavior of Ralstonia solanacearum race 3 biovar 2 during latent and active infection of geranium. Phytopathology 95:136-143.

32. Swofford, D. L. 2002. Phylogenetic Analysis Using Parsimony (*and Other Methods), 4.0 ed. Sinauer Associates, Sunderland, MA.

33. Taghavi, M., Hayward, A., Sly, L., and Fegan, M. 1996. Analysis of the phylogenic relationships of strains of Burkholderia solanacearum, Pseudomonas syzygii, and the blood disease bacterium of banana based on 16S rRNA sequences. Int. J. Syst. Bacteriol. 46:10-15.

34. Thurston, H. D. 1963. Bacterial wilt of potatoes in Colombia. Am. Potato J. 40:381-390.

35. Thwaites R., Mansfield, J., Eden-Green, S., and Seal, S. 1999. RAPD and rep PCR-based fingerprinting of vascular bacterial pathogens of Musa spp. Plant Pathol. 48:121-128.

36. United States Department of Agriculture (USDA). 2006. Agricultural Statistics, 1995-2006. National Agricultural Statistics Service.

37. United States Department of Agriculture (USDA-APHIS). 2004. Minimum sanitation protocols for offshore geranium cutting production.

38. United States Department of Commerce, Bureau of Census. 2004. World Trade Atlas, Global Trade Information Services.

39. Velupillai, M., and Stall, R. E. 1984. Variation among strains of Pseudomonas solanacearum from Florida. Proc. Fla. State Hortic. Soc. 97:209-213.

40. Villa, J. E., Tsuchiya, K., Horita, M., Opina, N., and Hyakumachi, M. 2005. Phylogenetic relationships of Ralstonia solanacearum species complex strains from Asia and other continents based on 16S rDNA, endoglucanase, and $h r p$ B gene sequences. J. Gen. Plant Pathol. 71:39-46.

41. Vos, P., Hogers, R., Bleeker, M., Reijans, M., van de Lee, T., Hornes, M., Frijters, A., Pot, J., Peleman, J., and Kuiper, M. 1995. AFLP: A new technique for DNA fingerprinting. Nucleic Acids Res. 23(21):4407-4414.

42. Williamson, L., Nakaho, K., Hudelson, B., and Allen, C. 2002. Ralstonia solanacearum race 3, biovar 2 strains isolated from geranium are pathogenic on potato. Plant Dis. 86:987-991. 